Herbert Prinzen · Ennius im Urteil der Antike 


\section{DRAMA}

\section{Beiträge zum antiken Drama und seiner Rezeption}

Herausgegeben von F. De Martino -

J. A. López Férez - G. Mastromarco -

B. Seidensticker - N. W. Slater -

A. H. Sommerstein - R. Stillers -

P. Thiercy - B. Zimmermann 


\author{
Beiheft 8
}

Herbert Prinzen

\title{
Ennius im Urteil der Antike
}

Verlag J. B. Metzler Stuttgart - Weimar 
D 61 (Dissertation der Heinrich-Heine-Uníversität Düsseldorf 1995)

Die Deutsche Bibliothek - CIP-Einheitsaufnahme

Prinzen, Herbert:

Ennius im Urteil der Antike / Herbert Prinzen. -

Stuttgart ; Weimar : Metzler, 1998

(Drama : Beiheft ; 8)

Zugl.: Düsseldorf, Univ., Diss., 1995

ISBN 978-3-476-45205-4

ISBN 978-3-476-45205-4

ISBN 978-3-476-04299-6 (eBook)

DOI 10.1007/978-3-476-04299-6

Dieses Werk ist einschließlich aller seiner Teile geschützt. Jede Verwertung außerhalb der engen Grenzen des Urheberrechtsgesetzes ist ohne Zustimmung des Verlages unzulässig und strafbar. Das gilt insbesondere für die Vervielfältigungen, Übersetzung, Mikroverfilmungen und Einspeicherung in elektronischen Systemen.

M \& P Schriftenreihe für Wissenschaft und Forschung

(C) 1998 Springer-Verlag GmbH Deutschland

Ursprünglich erschienen bei J.B. Metzlersche Verlagsbuchhandlung und Carl Ernst Poeschel Verlag GmbH in Stuttgart 1998 
Vetustas quidem nobis semper, si sapimus, adoranda est.

(Macr. Sat. III,14,2)

Dicebat Bernardus Carnotensis nos esse quasi nanos gigantium humeris insidentes, ut possimus plura eis et remotiora videre, non utique proprii visus acumine aut eminentia corporis, sed quia in altum subvehimur et extollimur magnitudine gigantea. ${ }^{1}$

John of Salisbury, Metalogicus III,4

(ed. C.C.J. WEBB, 1929, 136)

1 Zum Bild, das als erster Bernhard von Chartres $(+1124 / 30)$ gebraucht zu haben scheint, A. BUCK, Aus der Vorgeschichte der 'Querelle des anciens et des modernes' in Mittelalter und Renaissance, in: Bibliothèque d'Humanisme et Renaissance 20, 1958, 527-541, auch in: A. BUCK, Die humanistische Tradition in der Romania, Bad Homburg, 1968, 75-91 (Lit.: 78 A. 293; bes. J. DE GHELLINCK, Nani et gigantes, ALMA 18, 1945, 25-29); vgl. dens., Die Rezeption in den romanischen Literaturen der Renaissance, Berlin 1976, 228ff.; P. WEITMANN, Die Problematik des Klassischen als Norm und Stilbegriff, AuA 35, 1989, 150-186, bes, 155 mit A. 32 + 33; W. SUERBAUM, Tradition. Gedanken zur antiken Metaphorik kulturellen Wandels, in: J. GRUBER / F. MAIER (Hgg.), Humanismus und Bildung. Zukunftschancen der Tradition. Beiträge zur Bildungstheorie und zur Didaktik der Alten Sprachen, Bd. 2: Interpretation (Auxilia 28), Bamberg 1991, 61-77, bes. 64ff. Zu Johannes von Salisbury: G. MISCH, Johann von Salisbury und das Problem des mittelalterlichen Humanismus, Nachr. d. Ak. d. Wiss. in Gött., I. philol.-hist. Kl. Nr. 6, 1960, 231-357. 


\section{VORWORT}

Die vorliegende Arbeit ist die für die Publikation überarbeitete und teilweise aktualisierte Fassung meiner im Sommersemester 1995 von der Philosophischen Fakultät der Heinrich-Heine-Universität angenommenen Dissertation. Die Tätigkeit an einer Ganztagsschule seit Sommer 1995 ließ für die Überarbeitung leider weniger Zeit übrig, als ich mir gewünscht hätte. So konnte die in den letzten Jahren erschienene Literatur nur noch hier und da eingearbeitet oder zumindest angeführt werden. Der größte Teil der zur Verfügung stehenden Zeit wurde für die Umsetzung formaler Vorgaben für den Druck, für die Einfügung von Querverweisen sowie für die Erstellung der Indices verwendet.

Eine erste nähere Bekanntschaft mit dem Nachleben des Ennius machte ich im Jahre 1983 im Rahmen eines Ennius-Hauptseminars unter der Leitung von Herrn Prof. Dr. Reinhard Häußler. Mehr als ein kurzer Überblick über das vielschichtige und verzweigte Thema mit Berücksichtigung der wichtigsten Literatur konnte damals nicht gegeben werden, so daß ein Gefühl des Ungenügens zurückblieb. Die Beschränkung auf direkte Urteile über Ennius schloß die Behandlung von Autoren aus, bei denen sich zwar eine Vielzahl von Ennius-Zitaten und -Reminiszenzen fand, ein explizites Urteil aber fehlte. In einem zweiten Anlauf konnten die 'Wandlungen des antiken Ennius-Bildes' (Thema der Staatsarbeit [1994]) in den einzelnen literarischen Epochen akzentuiert werden. Zahlreiche Probleme ließen sich wegen zeitlicher Beschränkung nicht weiter verfolgen. Die Anregung von Herrn Prof. Dr. Häußler, einzelnen Problemen eingehender nachzuspüren und das gesammelte Material in einer Dissertation zu veröffentlichen, konnte im Herbst 1990 in die Tat umgesetzt werden, als Frau Prof. Dr. Ilona Opelt mich als wissenschaftlichen Mitarbeiter am Düsseldorfer Seminar für Klassische Philologie verpflichtete und mir damit die Möglichkeit einer intensiveren Auseinandersetzung mit dem Ennius-Urteil der Antike gab. Wiewohl mein Doktorvater auf der Einschränkung des Themas 'Ennius im Urteil der Antike' (statt schlichtweg 'Die antike Ennius-Rezeption') insistierte, glaubte ich den gesteckten Rahmen in dem Maße weiten zu müssen, in dem objektivierbare Wirkungsweisen und Wirkungsgrade die Annahme eines implizierten Urteils gestatten oder nahelegen. Zitate, Imitationen und Parodien, Allusion und motivliche Instrumentalisierung kommen auch ohne direktes Urteil zum Zuge, soweit sie als signifikant betrachtet werden können. 
Nachhaltige Dankbarkeit gilt meinen unvermittelt verstorbenen Lehrern, Frau Prof. Dr. Ilona Opelt und Herrn St.-Prof. Dr. Wolfgang Heimbecher. Herrn Prof. Dr. Wolfram Ax schulde ich Dank furr die Verlängerung meines Dienstvertrages, für die Übernahme des Korreferates sowie für wertvolle Hinweise und weiterführende Ratschläge. Ebenso danke ich den Herren Professoren G. Binder und B. Effe für die Ehre, beim Philologischen Colloquium der Seminare für Klassische Philologie der Universitäten Bielefeld-Bochum-Düsseldorf im Mai 1995 einen Vortrag über 'Cicero und Ennius' halten zu dürfen. Für einzelne Hinweise zu Dank verpflichtet fühle ich mich ferner den Herren Professoren R. Glei, M. Vielberg und O. Skutsch, der mir die Güte erwies, meine Staatsarbeit zu lesen. Weiterhin danke ich dem Herausgeber, Herrn Prof. Dr. Bernhard Zimmermann, für die Aufnahme der Arbeit in die Reihe 'Drama'. Wieviel Dank ich meinem verehrten Lehrer und Doktorvater, Herrn Prof. Dr. Reinhard Häußler, schulde, läßt sich mit Worten nicht ausdrücken. Ohne seine Fürsprache und Vermittlung, ohne seinen stetigen ermunternden $\mathrm{Zu}-$ spruch und ohne seine konstruktive Kritik, ohne die Übermittlung ungezählter Hinweise und Lesefrüchte hätte die Arbeit weder in der vorliegenden Form noch überhaupt entstehen können.

Düsseldorf, im Mai 1998

H. P. 


\section{A. EINLEITUNG}

B. DER DRAMATIKER ENNIUS 13

I. DER KOMIKER ENNIUS 14

1. Ennius als auctor contaminationis bei Terenz $\quad 15$

2. Ennius im Komikerkanon des Volcacius Sedigitus 18

II. DER TRAGIKER ENNIUS $\quad 21$

1. Parodia Enniana bei Plautus und Terenz 21

2. Griechische Vorlage und lateinische Übertragung 28

3. Ennius' Stellung innerhalb der römischen Tragikertrias 33

4. Andromacha-Medea - Iphigenia 40

a. Spätere Bearbeitungen 40

b. Direkte Urteile und Anspielungen 42

c. Acherusia templa $\quad 50$

d. Der Medea-Prolog und die Rhetorik 58

e. Der Medea-Prolog bei Catull, Vergil und Ovid 63

f. Andromachas und Medeas Klagen 71

g. Rückblick und Ausblick:
Ennius-Zitate und -Reminiszenzen als indirekte Urteile

C. DER EPIKER ENNIUS 91

I. DER DICHTER DER RÖMER IN REPUBLIKANISCHER ZEIT: HOMERUS ALTER UND PRIMUS DISCIPULUS MUSARUM $\quad 94$

1. Plautus: Annalen-Parodie? 96

2. Lucilius: Ennius - Homerus alter ?! 98

a. Satura - saturae - Literaturkritik 98

b. Concilium deorum 102

c. Mutando ridere: horret et - alget 108

d. Annales Enni als poesis 113

e. Enniana und Luciliana:
Anerkennung des Epos - Rechtfertigung der Satire
117

f. Zusammenfassung 124

3. Porcius Licinus und Pompilius:

$\begin{array}{ll}\text { Ennius Musarum }<\text { discipulus }> & 125\end{array}$ 
4. Lukrez: Dichtung und (Irr-)Lehre

a. Die Anerkennung des 'Dichters' Ennius 132

b. Die Ablehnung der homerisch-ennianischen Lehre $\quad 134$

c. Epikur - Ennius - Empedokles 138

d. Acherusia templa - lacus Averni 141

e. Ennius poeta - Ennius vates $\quad 145$

f. Zusammenfassung 147

5. Varro: Ennius - der Dichter der Römer 149

a. Ennius-Urteile und -Zitate in Varros Schriften 149

b. Ilias Homeri et Annales Enni 152

c. Ennius und die Musen 155

d. Testamentum 158

e. Zusammenfassung 160

6. Cicero: Ennius - Höhepunkt römischer Poesie 161

a. Ingeniosus poeta et auctor valde bonus 161

b. Ennius-Zitate zu Beginn einer Schrift oder eines Buches $\quad 171$

c. Livius Andronicus - Naevius - Ennius (Cic. Brut. 70ff.) 176

d. Somnium Ennii - Somnium Scipionis 182

e. Zusammenfassung 184

7. Cantores Euphorionis und Catull: Epyllion contra Epos 186

a. Die Problematik eines indirekten Ennius-Urteils bei Catull 186

b. Cantores Euphorionis 186

c. Catull c. 1: Widmung und Programm 189

d. Catull und die Ennius-Epigonen 194

e. Parodia Enniana 201

f. Rückblick und Ausblick 203

II. PATER ENNIUS UND DIE DICHTER DER AUGUSTEISCHEN ZEIT: INGENIO MAXIMUS, ARTE RUDIS

1. Vergil: Non Homerus alter, sed primus Homerus apud Latinos

a. Aurum in stercore quaero 213

b. Vergil zwischen Ennius und Homer 215

c. Vergil - Ennius - Naevius: Konzeptionelle Anregungen und color Ennianus $\quad 219$

d. Maior rerum mihi nascitur ordo; maius opus moveo 226

e. Römisches Sendungsbewußtsein bei Ennius 229

f. Fidus Achates und der Vertraute des Servilius:

g. Zusammenfassung 244 
2. Horaz: [Pater] Ennius et sapiens et fortis et [non] alter Homerus

a. Horaz über Ennius (und Lucilius): rudis et Graecis intacti carminis auctor - disiecti membra poetae

b. Pater Ennius - alter Homerus

c. Zusammenfassung

3. Pater Ennius in der Recusatio des Callimachus Romanus

a. Pater Ennius - Respekt oder Ironie?

b. Ennius hirsuta cingat sua dicta corona

c. Zusammenfassung

4. Ovid: Ennius ingenio maximus, arte rudis

a. Ennius-Reminiszenzen bei Ovid 270

b. Ovid über Ennius und Lukrez 271

c. Ingeniosi poetae 274

d. Annales Enni: nihil est hirsutius illis 281

e. Rückblick 283

III. ENNIUS UND DIE RÖMISCHE GESCHICHTSSCHREIBUNG DER REPUBLIKANISCHEN UND AUGUSTEISCHEN ZEIT:

CATO, COELIUS, SALLUST UND LIVIUS

1. Cato und Ennius 289

a. Von Sardinien nach Rom 289

b. Familiaris noster Ennius - Ennius, Lagerpoet des Fulvius 294

c. Annales und Origines 297

d. Zusammenfassung 301

2. Q. Ennius eumque studiose aemulatus L. Coelius 302

a. Archaismen und Poetismen 302

b. Wortstellung 307

c. Hannibal in somnis a Iove in deorum concilium vocatus 309

d. Zusammenfassung 313

3. Hexametrische Gebilde bei Sallust, Livius und Tacitus 315
a. Methodische Überlegungen
b. Sallust
c. Livius
d. Tacitus
e. Zusammenfassung 
IV.VON TIBERUS BIS DOMITIAN:

WECHSELHAFTE ZEITEN

A. TIBERISCH-CLAUDISCHE ZEIT:

ERSTARRUNG UND NEUES LEBENSGEFÜHL

Exkurs: Ennius bei Lucan

1. Velleius Paterculus: Schweigen des Historikers oder Opfer der Überlieferung?

2. Valerius Maximus: Scipio und sein praeco rudis 342

3. Seneca: Maximus poetarum Ennius? 346

a. Gellius und Senecas Kritik an Ennius, Cicero und Vergil 346

b. Maximus poetarum Ennius? 358

c. Zusammenfassung 361

4. Persius: Schein und Sein - Anspruch und Nichterfuillung 362

a. Persius und der Homerus alter 362

b. Der Prolog des Persius - Eine Musen-Begegnung bei Ennius? 365

c. Rückblick

373

B. FLAVISCHE ZEIT:

KLASSIZISMUS CONTRA MODERNISMUS UND 'ARCHAISMUS'

1. Statius: Musa rudis ferocis Enni

2. Quintilian: Ennium sicut sacros vetustate lucos adoremus

3. Martial: Attonitusque legis 'terrai frugiferai'

4. Silius: Hic canet illustri primus bella Itala versu/ 390 attolletque duces caelo
a. (In)direkte Ennius-Benutzung durch Silius?
b. Das Ennius-Medaillon
Exkurs: Ennius als praeco virtutis
c. Rückblick

V. DIE ARCHAISTISCHE STRÖMUNG IM 2. NACHCHRISTLICHEN JAHRHUNDERT:

QUINTUS ENNIUS NOSTER REDIVIVUS

1. Fronto: Quintus Ennius noster - Bindeglied zwischen

Lehrer und Schüler

2. Gellius: Ennius und die Philosophen 
1. Macrobius: Quantum Vergilius noster ex antiquiorum lectione profecerit

2. Claudian (und Petrarca): Gaudet enim virtus testes sibi iungere Musas

\section{APPENDICES}

I. Sentenzen und Wortspiele bei Ennius Wortspiele mit Ennius

II. Ennianisches im Geburtstagsgedicht Tibulls?

ZUSAMMENFASSUNG

INDICES

I. STELLENREGISTER

II. ENNIUS IM URTEIL DER ANTIKE

A. Ennius bei antiken Autoren:

Reminiszenzen - Zitate - Urteile

B. Urteile

III. NAMEN- UND SACHEN 